$\mathrm{MZ}-\mathrm{TH} / 00-28$

hep-th/0012146

Nov 202000

\title{
Exact solutions of Dyson-Schwinger equations for iterated one-loop integrals and propagator-coupling duality
}

\author{
D. J. Broadhurst ${ }^{1)}$ and D. Kreimer ${ }^{2)}$
}

\begin{abstract}
The Hopf algebra of undecorated rooted trees has tamed the combinatorics of perturbative contributions, to anomalous dimensions in Yukawa theory and scalar $\phi^{3}$ theory, from all nestings and chainings of a primitive self-energy subdivergence. Here we formulate the nonperturbative problems which these resummations approximate. For Yukawa theory, at spacetime dimension $d=4$, we obtain an integrodifferential DysonSchwinger equation and solve it parametrically in terms of the complementary error function. For the scalar theory, at $d=6$, the nonperturbative problem is more severe; we transform it to a nonlinear fourth-order differential equation. After intensive use of symbolic computation we find an algorithm that extends both perturbation series to 500 loops in 7 minutes. Finally, we establish the propagator-coupling duality underlying these achievements making use of the Hopf structure of Feynman diagrams.
\end{abstract}

1) D.Broadhurst@open.ac.uk; http://physics.open.ac.uk dbroadhu Physics Dept, Open University, Milton Keynes MK7 6AA, UK

$\left.{ }^{2}\right)$ Dirk.Kreimer@uni-mainz.de; http://dipmza.physik.uni-mainz.de/kreimer/homepage.html Heisenberg Fellow, Physics Dept, Univ. Mainz, 55099 Mainz, Germany 


\section{Introduction}

In [1, 2] we developed the Hopf-algebra techniques of [3, 4, 5, 6] to tame the combinatoric explosion of perturbation theory. For Yukawa theory at spacetime dimension $d=4$, and also for a scalar $\phi^{3}$ theory at its critical dimension $d=6$, we resummed all nestings and chainings of a divergent self-energy skeleton, using the Hopf algebra of undecorated rooted trees, to progress beyond the rainbow [7, 8] and chain [9, 10] approximations for anomalous dimensions. Padé-Borel resummation to 30 loops gave stable results at a Yukawa coupling $g=30$, enabling comparison with resummation methods. However, our previous work left unanswered two pertinent questions.

1. What are the nonperturbative problems whose perturbations [1, 2] were developed?

2. Are there limits on the coupling strength for which resummation may be performed?

Here we answer both questions. Our results provide a stringent testing ground [11] for confronting resummations of symbolically computed perturbation theory with high-precision numerical analyses of the corresponding nonperturbative integrodifferential equations.

Sect. 2 derives the nonperturbative problems as integrodifferential Dyson-Schwinger equations. These may be transformed to nonlinear differential equations, of second order in the Yukawa case and fourth order in the scalar case. The anomalous dimension is then defined, nonperturbatively, by the unique value of the derivative of the renormalized selfenergy at the subtraction point $q^{2}=\mu^{2}$ that guarantees a well-defined self-energy at all euclidean momenta with $0<q^{2}<\mu^{2}$. For the Yukawa case a parametric solution is obtained in terms of the complementary error function, erfc, with the anomalous dimension $\widetilde{\gamma}$ at coupling $a=(g / 4 \pi)^{2}$ given by the implicit condition

$$
\sqrt{\frac{a}{2 \pi}}=\exp \left(p_{0}^{2}\right) \operatorname{erfc}\left(p_{0}\right) ; \quad p_{0}:=\frac{\widetilde{\gamma}+2}{\sqrt{2 a}}
$$

which we solve by a Newton-Raphson method. The asymptotic perturbation series

$$
\widetilde{\gamma} \simeq \sum_{n>0} \widetilde{G}_{n} \frac{(-a)^{n}}{2^{2 n-1}}
$$

has a coefficient at $n+1$ loops given recursively by

$$
\widetilde{G}_{n+1}=\delta_{n, 0}+n \sum_{k=1}^{n} \widetilde{G}_{k} \widetilde{G}_{n+1-k} .
$$

The corresponding problem in the scalar theory entails boundary conditions on a nonlinear fourth-order differential equation. We show how to develop the perturbation series

$$
\gamma \simeq \sum_{n>0} G_{n} \frac{(-a)^{n}}{6^{2 n-1}}
$$

for the scalar anomalous dimension $\gamma$, far beyond the 30 loops obtained in [1]. After attempting a variety of methods, we found one that delivers 500 loops in 7 minutes, giving the 1675-digit integer $G_{500}$ of Table 1 . 
In the Yukawa case, an obstacle exists at the critical coupling constant $g_{\text {crit }}:=2(2 \pi)^{3 / 2} \approx$ 31.5 , corresponding to a critical anomalous dimension $\widetilde{\gamma}_{\text {crit }}:=-2$. The asymptotic series

$$
\widetilde{\gamma} \simeq 2 \sum_{n>0}(2 n-1) ! !\left(\frac{-a}{(\widetilde{\gamma}+2)^{2}}\right)^{n}
$$

is obtained as $a \rightarrow 0$. It is clearly vacuous as $\widetilde{\gamma}$ approaches -2 . At such strong couplings, one should use our new nonperturbative result (1), with

$$
\operatorname{erfc}\left(p_{0}\right):=\frac{2}{\sqrt{\pi}} \int_{p_{0}}^{\infty} d p \exp \left(-p^{2}\right)=1-\frac{2 p_{0}}{\sqrt{\pi}} \sum_{n=0}^{\infty} \frac{\left(-p_{0}^{2}\right)^{n}}{n !(2 n+1)} .
$$

Having thus solved the Yukawa problem at all couplings, we use its perturbative formulation to motivate methods for the more severe fourth-order scalar problem, whose Dyson-Schwinger equation indicates an obstacle at $\gamma=-1$.

\section{Dyson-Schwinger analyses}

In Yukawa theory, with an interaction term $g \bar{\psi} \sigma \psi$ at $d=4$, we consider the dimensionless renormalized self-energy term $\widetilde{\Sigma}\left(q^{2}\right)$ in the inverse propagator $\not\left(1-\widetilde{\Sigma}\left(q^{2}\right)\right)$ of a massless fermion field $\psi$ at euclidean 4-momentum $q$. The subtraction is performed at $q^{2}=\mu^{2}$, with $\widetilde{\Sigma}\left(\mu^{2}\right)=0$. Then $\widetilde{\gamma}$ is the value of $d \log \left(1-\widetilde{\Sigma}\left(q^{2}\right)\right) / d \log \left(q^{2}\right)$ at $q^{2}=\mu^{2}$. In the scalar theory at $d=6$ we consider an interaction $g \phi^{\dagger} \sigma \phi$ of the massless neutral scalar field $\sigma$ with a massless charged scalar field $\phi$, whose inverse propagator is $q^{2}\left(1-\Sigma\left(q^{2}\right)\right)$.

The infinite set of Feynman diagrams which we resum comprises all those whose subdivergences result from nestings and chainings of the one-loop self-energy skeleton. A 12-loop example is given in Fig. 1, with a subdivergence structure encoded by the undecorated rooted tree of Fig. 2. The Hopf algebraic methods of [2] gave the integer coefficients $\widetilde{G}_{n}$ and $G_{n}$ of the perturbation series (2, 4) up to $n=12$ loops, with a coupling $a:=g^{2} /(4 \pi)^{d / 2}$. In [1] we were able to extend this analysis to 30 loops, obtaining

$$
\begin{aligned}
& \widetilde{G}_{30}=10272611586206353744425870217572111879288 \\
& G_{30}=19876558632009586773182109989526780486481329823560105761256963720
\end{aligned}
$$

each of which subsumes $4.6 \times 10^{20}$ subtractions in the BPHZ formalism [12]. At that point, our dimensionally regularized method hit a limited imposed by memory requirements, since we had to store a triple series in powers of the coupling $a$, the $\log \operatorname{arithm} \log \left(q^{2} / \mu^{2}\right)$, and a dimensional regularization parameter $\varepsilon$.

We shall greatly improve on the previous analyses [1, 2], by using Dyson-Schwinger methods. First we show how to reduce the triple series to a double series, by dispensing with dimensional regularization. Then we describe the symbolic computation proof methods that led to discovery of a single-series method, which dispenses with the logarithm. They culminate in a propagator-coupling duality which we then prove thanks to the Hopf algebra structure. 


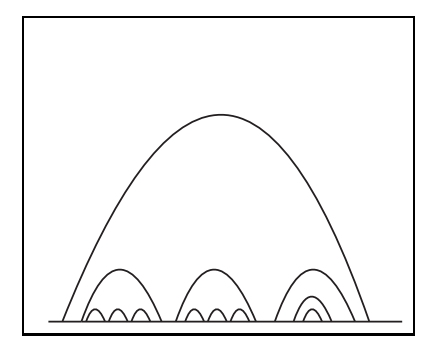

Figure 1: A twelve-loop example of the type of diagrams considered.

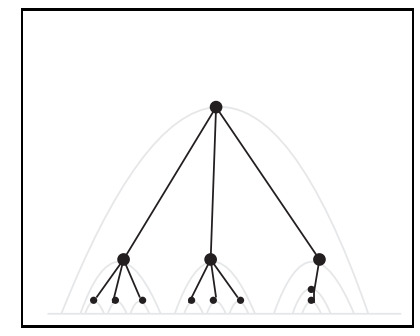

Figure 2: The corresponding tree structure which determines the necessary subtractions to get local counterterms.

\subsection{Integrodifferential equations}

Modulo divergences, the Yukawa problem corresponds to the Dyson-Schwinger equation

$$
q^{2} \widetilde{\Sigma}\left(q^{2}\right)=\frac{a}{\pi^{2}} \int \frac{d^{4} l}{l^{2}-l^{2} \widetilde{\Sigma}\left(l^{2}\right)} \frac{q \cdot l}{(q+l)^{2}}-\text { subtractions }
$$

with subtractions that give $\tilde{\Sigma}\left(\mu^{2}\right)=0$.

Let us pause for a moment and consider the structure of this equation. Its design guarantees that it represents a sum over all undecorated rooted trees: If we let

$$
q^{2} \widetilde{\boldsymbol{\Sigma}}\left(q^{2}\right)[t]=\frac{a}{\pi^{2}} \int d^{4} l \widetilde{\boldsymbol{\Sigma}}\left(l^{2}\right)\left[B_{-}(t)\right] \frac{q \cdot l}{l^{2}(q+l)^{2}}
$$

be, for each $q^{2}$, a character on the Hopf algebra of undecorated rooted trees (which implies to define $\widetilde{\boldsymbol{\Sigma}}\left(q^{2}\right)[e]:=1$ and $\left.\widetilde{\boldsymbol{\Sigma}}\left(q^{2}\right)\left[t_{1} t_{2}\right]:=\widetilde{\boldsymbol{\Sigma}}\left(q^{2}\right)\left[t_{1}\right] \widetilde{\boldsymbol{\Sigma}}\left(q^{2}\right)\left[t_{2}\right]\right)$, then we can regard

$$
\widetilde{B_{+}}:=\frac{a}{\pi^{2}} \int d^{4} l \frac{q \cdot l}{l^{2}(q+l)^{2}}
$$

as the corresponding representation of the $B_{+}$operator with coproduct $\Delta(T)=T \otimes 1+$ $\left(\mathrm{id} \otimes B_{+}\right) \Delta\left(B_{-}(T)\right)[2]$,

$$
\widetilde{B_{+}}(\widetilde{\mathbf{\Sigma}})\left(q^{2}\right)[t]:=\tilde{\mathbf{\Sigma}}\left(q^{2}\right)\left[B_{+}(t)\right]=\frac{a}{\pi^{2}} \int d^{4} l \tilde{\mathbf{\Sigma}}\left(l^{2}\right)[t] \frac{q \cdot l}{l^{2}(q+l)^{2}} .
$$

The Dyson-Schwinger equation can then be written as

$$
q^{2} \tilde{\boldsymbol{\Sigma}}\left(q^{2}\right)[X]=\frac{a}{\pi^{2}} \int d^{4} l \tilde{\boldsymbol{\Sigma}}\left(l^{2}\right)[1 /(1-X)] \frac{q \cdot l}{l^{2}(q+l)^{2}},
$$


where the series in Hopf algebra elements $X$ is defined by $X=a B_{+}(1 /(1-X))$, a form which we will use later on.

As we are confronting a character of the Hopf algebra of undecorated rooted trees, prominent in the study of differential equations in the context of Runge-Kutta methods, we expect to be able to find a solution to the Dyson-Schwinger equation turning it into a differential equation. This is indeed the case. We proceed by first turning the problem into a integrodifferential equation.

The integration over the 4-dimensional euclidean loop momentum, $l$, factors into an angular and a radial part. The angular integration of

$$
\frac{q \cdot l}{(q+l)^{2}}=\frac{1}{2}-\frac{q^{2}+l^{2}}{2(q+l)^{2}}
$$

is performed by using the 4-dimensional angular average

$$
q^{2} l^{2}\left\langle\frac{1}{(q+l)^{2}}\right\rangle_{d=4}=\min \left(q^{2}, l^{2}\right) .
$$

We split the radial integral over $y:=l^{2}$ into parts with $y<q^{2}$ and $y>q^{2}$. For the latter we fix the subtractions by requiring that $\widetilde{\Sigma}\left(\mu^{2}\right)=0$, obtaining the Dyson-Schwinger equation

$$
\begin{aligned}
\widetilde{\Sigma}\left(q^{2}\right) & =\frac{a}{2} \int_{\mu^{2}}^{q^{2}} \frac{d y}{y-y \widetilde{\Sigma}(y)}+\widetilde{F}\left(\mu^{2}\right)-\widetilde{F}\left(q^{2}\right) \\
\widetilde{F}(x) & :=\frac{a}{2} \int_{0}^{x} \frac{d y}{y-y \widetilde{\Sigma}(y)}\left(\frac{y}{x}\right)^{2} .
\end{aligned}
$$

Differentiating w.r.t. $x:=q^{2}$ we obtain the integrodifferential equation

$$
x^{3} \frac{d}{d x} \tilde{\Sigma}(x)=a \int_{0}^{x} \frac{y d y}{1-\widetilde{\Sigma}(y)}
$$

which makes no reference to the subtraction point.

To repeat this analysis in the scalar case, we use the 6-dimensional angular average

$$
q^{2} l^{2}\left\langle\frac{1}{(q+l)^{2}}\right\rangle_{d=6}=N-\frac{N^{3}}{3 q^{2} l^{2}} ; \quad N:=\min \left(q^{2}, l^{2}\right)
$$

which gives the Dyson-Schwinger equation

$$
\begin{aligned}
\Sigma\left(q^{2}\right) & =\frac{a}{6} \int_{\mu^{2}}^{q^{2}} \frac{d y}{y-y \Sigma(y)}+F\left(\mu^{2}\right)-F\left(q^{2}\right) \\
F(x) & :=\frac{a}{6} \int_{0}^{x} \frac{d y}{y-y \Sigma(y)}\left(\frac{y^{3}}{x^{3}}-3 \frac{y^{2}}{x^{2}}+3 \frac{y}{x}\right)
\end{aligned}
$$

and hence the integrodifferential equation

$$
x^{4} \frac{d}{d x} \Sigma(x)=\frac{a}{2} \int_{0}^{x} \frac{(x-y)^{2} d y}{1-\Sigma(y)}
$$


which likewise makes no reference to the subtraction point. We require the solution to (18) that is regular for $0<x \leq \mu^{2}$, with $\Sigma\left(\mu^{2}\right)=0$. Then the scalar anomalous dimension is

$$
\gamma:=\left.\frac{d \log \left(1-\Sigma\left(q^{2}\right)\right)}{d \log \left(q^{2}\right)}\right|_{q^{2}=\mu^{2}}=-\frac{a}{2} \int_{0}^{1} \frac{(1-r)^{2} d r}{1-\Sigma\left(\mu^{2} r\right)} .
$$

\subsection{Multiloop integers from nonlinear differential equations}

By differentiating (14) once and (18) three times we obtain

$$
\left(1-\tilde{\Sigma}\left(q^{2}\right)\right) \widetilde{P}(D) \widetilde{\Sigma}\left(q^{2}\right)=a=\left(1-\Sigma\left(q^{2}\right)\right) P(D) \Sigma\left(q^{2}\right)
$$

with polynomials of the differential operator $D:=d / d \log \left(q^{2}\right)$ given by

$$
\begin{aligned}
& \widetilde{P}(D):=D(D+2) \\
& P(D):=D(D+1)(D+2)(D+3)
\end{aligned}
$$

and already encountered in analyses of rainbow and chain contributions. The rainbow diagrams generate contributions to the anomalous dimensions that solve

$$
\widetilde{P}\left(-\widetilde{\gamma}_{\text {rainbow }}\right)=a=P\left(-\gamma_{\text {rainbow }}\right)
$$

namely [7, 8]

$$
\begin{aligned}
\widetilde{\gamma}_{\text {rainbow }}=1-\sqrt{1+a} & =-\frac{a}{2}+\frac{a^{2}}{2^{3}}-2 \frac{a^{3}}{2^{5}}+5 \frac{a^{4}}{2^{7}}+\cdots \\
\gamma_{\text {rainbow }}=\frac{3-\sqrt{5+4 \sqrt{1+a}}}{2} & =-\frac{a}{6}+11 \frac{a^{2}}{6^{3}}-206 \frac{a^{3}}{6^{5}}+\cdots
\end{aligned}
$$

whereas insertion of chains in the one-loop diagram gives [1]

$$
\begin{aligned}
& \widetilde{\gamma}_{\text {chain }}=-2 \int_{0}^{\infty} \exp (-2 x / a) \frac{x d x}{\widetilde{P}(x)} \simeq-\frac{a}{2}+\frac{a^{2}}{2^{3}}-2 \frac{a^{3}}{2^{5}}+6 \frac{a^{4}}{2^{7}}+\cdots \\
& \gamma_{\text {chain }}=-6 \int_{0}^{\infty} \exp (-6 x / a) \frac{x d x}{P(x)} \simeq-\frac{a}{6}+11 \frac{a^{2}}{6^{3}}-170 \frac{a^{3}}{6^{5}}+\cdots
\end{aligned}
$$

At $n<3$ loops, rainbow and chain contributions correspond to same rooted trees. At $n=3$ loops, there are 2 rooted trees and the full Hopf algebra gives the sum of the rainbow and chain contributions, with $\widetilde{G}_{3}=2+2=4$ and $G_{3}=206+170=376$. At $n>3$ loops, there are essentially new terms in the Hopf algebra. For example, $\widetilde{G}_{4}=27>5+6$ receives contributions from all 4 rooted trees with 4 nodes.

Thanks to the differential equations in (20) we can achieve a dramatic speedup of the dimensionally regularized methods in [1, 2]. The $n$-loop contribution to $\Sigma\left(q^{2}\right)$ has the form $a^{n} \sum_{k=1}^{n} C(n, k) L^{k}$, with $L:=\log \left(q^{2} / \mu^{2}\right)$. Substituting this Ansatz into the nonlinear fourth-order differential equation $a=\left(1-\Sigma\left(q^{2}\right)\right) P(D) \Sigma\left(q^{2}\right)$, we require that all powers of the log disappear on the r.h.s. and that only the first power of the coupling survives. 
Then the integer $G_{n}=6(-36)^{n-1} C(n, 1)$ is obtained by iterative solution of a system of equations that gives $n$ linear combinations of $\{C(n, k) \mid 1 \leq k \leq n\}$ in terms of $O\left(n^{3}\right)$ products of $\{C(m, k) \mid 1 \leq k \leq m<n\}$.

This Dyson-Schwinger algorithm may be implemented by the REDUCE procedure

procedure HopfScalar(n); for $m:=1: n$ do

$<$ s $m:=$ for $\mathrm{k}:=1: \mathrm{m}$ sum $\mathrm{c} k * \log \mathrm{x}^{\wedge} \mathrm{k}$;

$\mathrm{d} m:=$ if $\mathrm{m}=1$ then 1 else for $\mathrm{k}:=1: \mathrm{m}-1$ sum $\mathrm{d} k * \mathrm{~s}(\mathrm{~m}-\mathrm{k})$;

sol:=first solve coeff $(x * d f(x \wedge 3 * s m, x, 4)-d m, l o g x)$;

$\mathrm{s} \mathrm{m}:=$ if $\mathrm{m}=1$ then $\log \mathrm{x} / 6$ else ( $\mathrm{s} \mathrm{m}$ where $\mathrm{sol}$ );

write $\mathrm{G} \mathrm{m}:=6(-36)^{\wedge}(\mathrm{m}-1) * \operatorname{sub}(\mathrm{x}=1, \mathrm{df}(\mathrm{s} \mathrm{m}, \mathrm{x}))>>$;

which invokes the procedures solve, coeff and df, to solve for the coefficients of the results of differentiations. It yields the 30-loop coefficient (8) in 6 seconds, running version 3.7 [16] of REDUCE on a $500 \mathrm{MHz}$ alpha machine. By way of comparison, the dimensionally regularized 30-loop calculation in [1] took 8 hours, on the same machine. The origin of the speedup is clear: here we have only a double expansion, in the coupling and the $\operatorname{logarithm}$, whereas in [1, 2] we also had a Laurent series in $\varepsilon$, where the limit $\varepsilon \rightarrow 0$ was admissible only at the final stage of the dimensionally regularized BPHZ procedure. Now that we have a nonperturbative Dyson-Schwinger formulation, we can dispense with the BPHZ complexities.

Note that, even using the new method, the time to reach $n$ loops grows rather rapidly with $n$. At each iteration with $m \leq n$ we solve a system of $m$ linear equations and hence perform a number of elementary operations that grows like $m^{3}$. The main cost comes from $O\left(m^{3}\right)$ multiplication of integers with $O(m \log m)$ digits at the $m$-th step of the iteration. It follows that the time to reach $n$ loops scales like $n^{c}$, modulo logarithms of $n$, with an exponent $c \geq 5$, in any implementation, and with $c \leq 6$, in any sensible implementation. To achieve the lower bound $c=5$ one might need to use fast Fourier transforms. We devised REDUCE code, more structured than that above, whose timing scaled, empirically, with an exponent $c \approx 5.3$, for $n=O(100)$. After a couple of days we reached 420 loops and checked the integer $G_{420}$ by independent code that used David Bailey's MPFUn package [17]. As might have been expected, Bailey's multiple-precision extension of FORTRAN was somewhat faster. Table 1 gives the result for $G_{500}$, obtained by running MPFUN for 21.5 hours.

We now chart the discovery of a second dramatic speedup, which reduces the number of multiplications of large integers at the $m$-th step from $O\left(m^{3}\right)$ to merely $O(m)$.

\subsection{Parametric solution of the Yukawa problem}

For the Yukawa problem we work with the variable $z:=\left(q^{2} / \mu^{2}\right)^{2}$ and define

$$
\widetilde{G}(z):=\sqrt{2 / a}\left(z-z \widetilde{\Sigma}\left(\mu^{2} z^{1 / 2}\right)\right)
$$


normalized so that $\widetilde{G}(1)=\sqrt{2 / a}$. Then the differential equation is simply

$$
2 \widetilde{G} \widetilde{G}^{\prime \prime}=-1
$$

where primes denote differentiation w.r.t. $z$. This is easily integrated, to give

$$
\left(\widetilde{G}^{\prime}\right)^{2}=-\log \widetilde{G}+\text { constant }
$$

The next step is to work with the parameter $p:=\widetilde{G}^{\prime}$, in terms of which

$$
\widetilde{G}=\sqrt{2 / a} \exp \left(p_{0}^{2}-p^{2}\right)
$$

where $p_{0}$ is the value of $p$ for which $z=1$ and hence $q^{2}=\mu^{2}$. Then a parametric solution is obtained by determining

$$
\widetilde{\alpha}(p):=z / \widetilde{G}
$$

which satisfies the first-order equation

$$
\widetilde{\alpha}=\frac{1}{p}+\frac{1}{2 p} \frac{d \widetilde{\alpha}}{d p}
$$

whose iterative solution gives the asymptotic series

$$
\widetilde{\alpha}(p) \simeq \frac{1}{p}+\frac{1}{p} \sum_{n>0} \frac{(2 n-1) ! !}{\left(-2 p^{2}\right)^{n}} .
$$

From (32) and the derivative of (28) at $z=1$, we determine

$$
\widetilde{\alpha}\left(p_{0}\right)=\sqrt{\frac{a}{2}} ; \quad p_{0}:=\frac{\widetilde{\gamma}+2}{\sqrt{2 a}}
$$

and hence obtain the asymptotic series (5) at $p=p_{0}$.

As advertised in the introduction, we can now give the complete nonperturbative solution of the $d=4$ case, by noting that the asymptotic series (34) is that of

$$
\widetilde{\alpha}(p)=\sqrt{\pi} \exp \left(p^{2}\right) \operatorname{erfc}(p)=2 \int_{p}^{\infty} d s \exp \left(p^{2}-s^{2}\right)
$$

Tidying up, we then obtain the parametric solution

$$
\begin{aligned}
\tilde{\Sigma}\left(q^{2}\right) & =1-\frac{\sqrt{a / 2 \pi}}{\exp \left(p^{2}\right) \operatorname{erfc}(p)} \\
q^{2} & =\mu^{2}\left(\frac{\operatorname{erfc}(p)}{\operatorname{erfc}\left(p_{0}\right)}\right)^{1 / 2}
\end{aligned}
$$

with the condition (四) determining the anomalous dimension, even in the strong-coupling regime $g>g_{\text {crit }}:=2(2 \pi)^{3 / 2} \approx 31.5$ where $a>2 \pi$ and hence $p_{0}:=(\widetilde{\gamma}+2) / \sqrt{2 a}<0$. 
Finally we obtain the elegant recursion (3) for the coefficients of the perturbation series (2), by turning (33), at $p=p_{0}$, into a nonlinear differential equation for the anomalous dimension. The result is conveniently written as

$$
2 \widetilde{\gamma}=-a+a^{2} \frac{d}{d a} \frac{\widetilde{\gamma}^{2}}{a}
$$

which proves (3) and generates the 500-loop Yukawa coefficient in 10 seconds. We remind the reader that this sums all diagrams at the 500-loop level generated by our DysonSchwinger equations, of which there are as many as there are rooted trees with 500 vertices. Finally, we note that at $\mathrm{g}=30$ the nonperturbative result for $\tilde{\gamma}$, to 120 digits, is

$-1.8520278058795936576120591680015771764936420535922291037938512550071476$ 394977783846264445624911569322962723432940741627702 ,

obtained by a Newton-Raphson method and in excellent agreement with resummation techniques [11].

\subsection{Asymptotic parametric solution of the scalar problem}

In the scalar case, at $d=6$, we work with the variable $y:=q^{2} / \mu^{2}$ and define

$$
G(y):=\sqrt{6 / a}\left(y-y \Sigma\left(\mu^{2} y\right)\right)
$$

which transforms the fourth-order equation for $\Sigma$ to

$$
G G^{\prime \prime}+\frac{G}{6} \frac{d}{d y}\left(5+y \frac{d}{d y}\right) y G^{\prime \prime}=-1
$$

where primes denote differentiation w.r.t. $y$. Taking $p:=G^{\prime}$ as the parameter, we define

$$
\alpha(p):=y / G ; \quad \beta(p):=-G G^{\prime \prime}
$$

with the formal solution

$$
\alpha(p)=\int_{p}^{\infty} \frac{d s}{\beta(s)} \exp \left(-\int_{p}^{s} \frac{t d t}{\beta(t)}\right)
$$

generalizing (36), which was the Yukawa case $\widetilde{\beta}=1 / 2$. The differential equations

$$
\begin{aligned}
& \alpha=\frac{1}{p}+\frac{\beta}{p} \frac{d \alpha}{d p} \\
& \beta=1+\frac{\beta}{6} \frac{d}{d p}\left(5-\alpha \beta \frac{d}{d p}\right) \alpha \beta
\end{aligned}
$$

may be solved iteratively, to yield the asymptotic series

$$
\begin{aligned}
& \alpha(p) \simeq \frac{1}{p}+\frac{6}{p} \sum_{n>0} \frac{A_{n}}{\left(-6 p^{2}\right)^{n}} \\
& \beta(p) \simeq 1+\sum_{n>0} \frac{B_{n}}{\left(-6 p^{2}\right)^{n}}
\end{aligned}
$$


where $A_{n}$ and $B_{n}$ are positive integers, with $A_{1}=1$ and $B_{1}=5$, obtained by inspection, leading to $A_{2}=23$ and $B_{2}=4 \times 43$, and so on. Diligent symbolic programming reduces the main computational burden to $O(n)$ multiplications of large integers, at the $n$-th iteration of (44,45).

By this means we developed 500 terms of the asymptotic series

$$
\gamma \simeq 6 \sum_{n>0} A_{n}\left(\frac{-a}{(6 \gamma+6)^{2}}\right)^{n}
$$

in a minute, while a day was needed to compute $G_{500}$, by the double-series method.

\subsection{Differential equation for the scalar anomalous dimension}

In the Yukawa case, we succeeded in computing $\widetilde{G}_{n}$ via $O(n)$ large-integer multiplications in (3). Similarly, in the scalar case, we programmed the iterations of (44,45) so as to obtain $A_{n}$ via $O(n)$ multiplications at the $n$-th step.

Yet there is a glaring discrepancy between the Yukawa and scalar analyses, thus far. The procedure for obtaining $G_{n}$ from $A_{n}$ appears to involve $O\left(n^{3}\right)$ multiplications. Thus we have not yet improved on the double-series method, in the scalar case. We eventually remedied this problem, by a sequence of computer-algebra explorations and finally reduced it to the procedure DysonScalar to which we now turn.

First, we sought generalizations of the method of (44,45). The factors of (22) reveal that so far we have exploited only one of three possibilities. More generally, we obtained

$$
\begin{aligned}
\alpha_{k} & =\frac{1}{p}+\frac{\beta_{k}}{p} \frac{d \alpha_{k}}{d p} \simeq \frac{1}{p}+\frac{6}{p} \sum_{n>0} \frac{A_{n, k}}{\left(-6 p^{2}\right)^{n}} \\
\beta_{k} & =1+\frac{\beta_{k}}{6} \frac{d}{d p}\left(11 k-6-k^{3} \alpha_{k} \beta_{k} \frac{d}{d p}\right) \alpha_{k} \beta_{k} \\
\gamma & \simeq 6 k \sum_{n>0} A_{n, k}\left(\frac{-k a}{(6 \gamma+6 k)^{2}}\right)^{n}
\end{aligned}
$$

which is valid for $k=1,2,3$, but for no other value. The analysis in (44,45,48) corresponds to the case with $k=1$.

To check that (50) carries the same information for $k=1,2,3$, we used computer algebra to transform it to a nonlinear third-order differential equation for $\gamma$, invoking the constraint $(k-1)(k-2)(k-3)=0$ to obtain

$$
\begin{aligned}
& 8 a^{3} \gamma\left\{\gamma^{2} \gamma^{\prime \prime \prime}+4 \gamma \gamma^{\prime} \gamma^{\prime \prime}+\left(\gamma^{\prime}\right)^{3}\right\}+4 a^{2} \gamma\left\{2 \gamma(\gamma-3) \gamma^{\prime \prime}+(\gamma-6)\left(\gamma^{\prime}\right)^{2}\right\} \\
& +2 a \gamma\left(2 \gamma^{2}+6 \gamma+11\right) \gamma^{\prime}-\gamma(\gamma+1)(\gamma+2)(\gamma+3)=a
\end{aligned}
$$

where the primes denote differentiation w.r.t. the coupling $a$. This seemingly baroque equation then remedies the previous discrepancy between the scalar and Yukawa cases, 
allowing an iterative determination of the coefficients $G_{n}$ via $O(m)$ large-integer multiplications at each $m \leq n$. The iterative procedure is based on the rearrangement

$$
\begin{aligned}
& 6 \gamma=-a+11 a^{2} \frac{d}{d a} \frac{\gamma^{2}}{a}-6 T_{3}+T_{4} \\
& T_{3}:=4 a^{2} \gamma\left\{\gamma \gamma^{\prime \prime}+\left(\gamma^{\prime}\right)^{2}\right\}-2 a \gamma^{2} \gamma^{\prime}+\gamma^{3} \\
& T_{4}:=8 a^{3} \gamma\left\{\gamma^{2} \gamma^{\prime \prime \prime}+4 \gamma \gamma^{\prime} \gamma^{\prime \prime}+\left(\gamma^{\prime}\right)^{3}\right\}+4 a^{2} \gamma^{2}\left\{2 \gamma \gamma^{\prime \prime}+\left(\gamma^{\prime}\right)^{2}\right\}+4 a \gamma^{3} \gamma^{\prime}-\gamma^{4}
\end{aligned}
$$

where the first two terms on the r.h.s. of (53) parallel those in (39) and the remainder are grouped in $T_{3}$, which is cubic in $\gamma$, and $T_{4}$, which is quartic.

At first sight, it was hard to spot the pattern of higher-order terms in (54,55). It emerged when we discovered that

$$
T_{3}=\gamma\left(2 a \frac{d}{d a}-1\right) T_{2} ; \quad T_{2}:=a^{2} \frac{d}{d x} \frac{\gamma^{2}}{a}=\gamma\left(2 a \frac{d}{d a}-1\right) \gamma
$$

It was then natural to suppose that

$$
T_{4}=\gamma\left(2 a \frac{d}{d a}-1\right) T_{3}
$$

and to verify that this indeed gives (55). Hence we achieve a highly systematic iteration

procedure DysonScalar $(\mathrm{n})$; for $\mathrm{m}:=1: \mathrm{n}$ do

$<<$ for $j:=1: 3$ do $g(j+1, m):=$ for $k:=j: m-1$ sum $g(m-k) *(2 k-1) * g(j, k)$;

write $G \mathrm{~m}:=11 \mathrm{~g}(2, \mathrm{~m}) / 6-6 \mathrm{~g}(3, \mathrm{~m})+6 \mathrm{~g}(4, \mathrm{~m})+$ if $\mathrm{m}=1$ then $1 ; \mathrm{g}(1, \mathrm{~m}):=6 \mathrm{G} \mathrm{m}>>$;

which generates the 500-loop integer of Table 1 in merely 7 minutes. We will derive this procedure from the Hopf algebra structure of the perturbation series $X$, but first we exhibit a further consequence of this iteration.

\section{Momentum dependence via the anomalous dimen- sion}

\subsection{Propagator-coupling duality}

Finally we arrive at a fine duality, between the dependence of the propagator

$$
\Pi\left(q^{2}\right):=\frac{1}{q^{2}-q^{2} \Sigma\left(q^{2}\right)}
$$

on the $\operatorname{logarithm} L:=\log \left(q^{2} / \mu^{2}\right)$, and the dependence of the (suitably normalized) coupling constant $\sqrt{a}:=g /(4 \pi)^{d / 4}$ on the dual of $L$, say $\lambda$, which we shall now identify. 
First consider the Dyson-Schwinger equation for $\Pi\left(q^{2}\right)$, which may be written

$$
P\left(q^{2} \frac{d}{d q^{2}}\right) \frac{1}{\Pi\left(q^{2}\right) q^{2} \sqrt{a}}=-\Pi\left(q^{2}\right) q^{2} \sqrt{a} .
$$

Now consider the apparently baroque third-order equation (52) for the anomalous dimension. Its origin is far simpler:

$$
P\left(-2 a \gamma(a) \frac{d}{d a}\right) \frac{1}{\sqrt{a}}=-\sqrt{a}
$$

with the same polynomial, of a different operator. Thus the duality is as follows.

Propagator-coupling duality At fixed coupling $a_{0}$ let

$$
\Pi\left(q^{2}\right) q^{2} \sqrt{a_{0}}=S(L) ; \quad L:=\log \left(q^{2} / \mu^{2}\right) .
$$

Let $\gamma(a)$ be the anomalous dimension at arbitrary coupling $a$. Then

$$
\sqrt{a}=S(\lambda) ; \quad \lambda:=\int_{a}^{a_{0}} \frac{d b}{2 b \gamma(b)} .
$$

Thus we expose the physical reasons for the enormous speedups that we have gained. The triple expansion in $a, L$ and $\varepsilon$, employed in [1, 2], is obviously reducible to a double expansion in $a$ and $L$, since we dispense with dimensional regularization, by taking derivatives of the Dyson-Schwinger equation. What was not so obvious, at the outset, is that the double expansion in $a$ and $L$, underlying the procedure HopfScalar of sect. 2.2, is also unnecessary. Now, thanks to the amazingly compact procedure DysonScalar of sect. 2.5, we have a procedure for the perturbation series of $\gamma(a)$ by iterative solution of the momentum-independent equation (60).

We had a strong intuition of the possibility of this second speedup, by a huge factor of $O\left(n^{2}\right)$ at $n$ loops, arguing as follows. Suppose that we knew the momentum dependence of the self energy, $\Sigma\left(q^{2}\right)$. Then the anomalous dimension would be known, from the derivative of the self energy at $q^{2}=\mu^{2}$, where $\Sigma\left(\mu^{2}\right)=0$. Conversely, one expects that a knowledge of the dependence of $\gamma(a)$ on the coupling $a$ is sufficient to determine the momentum dependence of the self energy, and hence that it is unnecessary to expand in both $a$ and $\log \left(q^{2} / \mu^{2}\right)$. The dictionary (61, 62) establishes this fact.

In summary: the self energy must be renormalized; the anomalous dimension encodes the infinities that have been subtracted. From the momentum dependence of the self energy one can find the anomalous dimension; in massless theories the converse is true. Note that we can read the duality as saying

$$
\log \left(q^{2} / \mu^{2}\right)=\int_{q^{2} \Pi\left(q^{2}\right)}^{1} \frac{d x}{x} \frac{1}{\gamma\left(x^{2} a\right)}
$$

or as

\section{Theorem 1:}

$$
\frac{d \log (1-\Sigma)}{d \log q^{2}}=\gamma\left(a /(1-\Sigma)^{2}\right)
$$

We now derive this result rigorously. 


\subsection{Proof of the duality}

We have to prove Theorem 1. We will rely on two propositions. The first exhibits how the Hopf and Lie algebra structure of perturbation theory interferes with the equation of motion - the Dyson-Schwinger equation- which is the defining equation $X=a B_{+}(1 /(1-$ $X)$ ) for the Hopf algebra element $X$. The second proposition establishes the recursion DysonScalar.

Let $X=\sum_{k=1}^{\infty} a^{k} X_{k}$ be a formal series in the Hopf algebra $H$ of undecorated rooted trees, such that the counit $\bar{e}$ vanishes, $\bar{e}(X)=0$, with $X_{k} \in H$ and $a$ be the coupling constant, and as announced above let $X$ be defined by

$$
X=a B_{+}(1 /(1-X)),
$$

where we write $1 /(1-X)=\sum_{j=0}^{\infty} X^{j}$. This determines the $X_{k} \in H$ uniquely. The first four $X_{k}$ read

$$
\begin{aligned}
& X_{1}=B_{+}(e) \\
& X_{2}=B_{+}\left(X_{1}\right) \\
& X_{3}=B_{+}\left(X_{2}\right)+B_{+}\left(X_{1} X_{1}\right) \\
& X_{4}=B_{+}\left(X_{3}\right)+2 B_{+}\left(X_{1} X_{2}\right)+B_{+}\left(X_{1} X_{1} X_{1}\right),
\end{aligned}
$$

which are linear combinations of rooted trees, and the unit $e$ of the algebra represents the empty tree.

Note that the weights in $X_{4}$ are not the Connes-Moscovici weights, but field-theoretic weights.

Let $F_{T}(U)$ be the befooting operator (with $F_{u_{1}}$ the usual befooting operator of [6, 21]) which sums over all ways of removing the tree $T$ from $U$, extended by linearity: for $Y=c T_{1}+d T_{2}$ a linear combination of rooted trees (ie an arbitrary element of the linear basis of $H$ ), let us set

$$
F_{Y}(U)=c F_{T_{1}}(U)+d F_{T_{2}}(U)
$$

Prop.1: The above coefficients $X_{i}$ of the series $X$ fulfill

$$
\left.F_{X_{m}}\left(X_{k}\right)=[2(k-m)-1)\right] X_{k-m}, k>m \geq 1 .
$$

Proof: The structure of the series $X$ gives the following form to the coefficients $X_{k}$ :

$$
X_{k+1}=B_{+}\left[\sum_{r=1}^{k} \sum_{\substack{s_{1} i_{1}+\ldots+s_{r} i_{r}=k \\ 0<i_{1}<\ldots<i_{r}}} X_{i_{1}}^{s_{1}} \ldots X_{i_{r}}^{s_{r}} \frac{r !}{s_{1} ! \ldots s_{r} !}\right], \forall k \geq 1 .
$$

Note that by definition $F_{X_{m}}\left(X_{k}\right)=0, m \geq k$ and that $F_{X_{m}}$ is a derivation, $F_{X_{m}}(U V)=$ $F_{X_{m}}(U) V+U F_{X_{m}}(V)$. Also,

$$
F_{X_{m}}\left(X_{m+1}\right)=X_{1}, \forall m \geq 1
$$


as

$$
\begin{aligned}
F_{X_{k}}\left(X_{k+1}\right) & =F_{X_{k}}\left(B_{+}\left[\sum_{r=1}^{k} \sum_{\substack{s_{1} i_{1}+\ldots+s_{r} i_{r}=k \\
0<i_{1}<\ldots<i_{r}}} X_{i_{1}}^{s_{1}} \ldots X_{i_{r}}^{s_{r}} \frac{r !}{s_{1} ! \ldots s_{r} !}\right]\right) \\
& =F_{X_{k}}\left(B_{+}\left(X_{k}\right)\right)=X_{1} .
\end{aligned}
$$

For any $m$ in $F_{X_{m}}\left(X_{i}\right)$, we assume the proposition holds for all $X_{i}, i \leq k$. We show that it holds for $X_{k+1}, \forall m$. For any $m$, Eq.(64) provides the start of the induction.

Now, $F_{X_{m}}$ and $B_{+}$have the commutator

$$
\left[F_{X_{m}}, B_{+}\right]\left(X_{k}\right)=\delta_{m, k} B_{+}(e)
$$

by the definition of $F_{X_{m}}$ ( $F_{X_{m}}$ removes feet, $B_{+}$grows at roots, only at $m=k$ this interferes in the indicated manner), which generalizes to any product $\prod_{i} X_{r_{i}}$ of elements $X_{r_{i}}$ so that the interchange of the befooting operator generates some derivatives of this product:

$$
F_{X_{m}} B_{+}\left[\prod_{i} X_{r_{i}}\right]=B_{+}\left[F_{X_{m}}\left(\prod_{i} X_{r_{i}}\right)+\sum_{j} \delta_{m, r_{j}} \prod_{i}^{\hat{r_{j}}} X_{r_{i}}\right],
$$

where the superscript $\hat{r}_{j}$ at the product indicates an omission of the factor $X_{r_{j}}$.

We can now interchange the action of the $B_{+}$and the $F_{X_{m}}$ operator in the expression for $F_{X_{m}}\left(X_{k+1}\right)$, and use the assumption to find the desired result. Let us work out an example before we give some more details.

$$
X_{6}=B_{+}\left[X_{5}+2 X_{1} X_{4}+2 X_{2} X_{3}+3 X_{1}^{2} X_{3}+3 X_{1} X_{2}^{2}+4 X_{1}^{3} X_{2}+X_{1}^{5}\right] .
$$

For $F_{X_{m}}\left(X_{6}\right)$ we thus find (using $F_{X_{m}}\left(X_{1}\right)=0, \forall m$ )

$$
\begin{aligned}
& B_{+}\left[F_{X_{m}}\left(X_{5}\right)+2 X_{1} F_{X_{m}}\left(X_{4}\right)+2 F_{X_{m}}\left(X_{2}\right) X_{3}+2 X_{2} F_{X_{m}}\left(X_{3}\right)+3 X_{1}^{2} F_{X_{m}}\left(X_{3}\right)\right. \\
& \left.+6 X_{1} X_{2} F_{X_{m}}\left(X_{2}\right)+4 X_{1}^{3} F_{X_{m}}\left(X_{2}\right)\right]+ \text { commutator terms. }
\end{aligned}
$$

To be specific, let us set $m=2$ to get, using the assumption of the induction,

$$
B_{+}\left[5 X_{3}+6 X_{1} X_{2}+2 X_{2} X_{1}+3 X_{1}^{2} X_{1}\right]
$$

while the commutator terms deliver

$$
B_{+}\left[2 X_{3}+6 X_{1} X_{2}+4 X_{1}^{3}\right] .
$$

Altogether, we get

$$
B_{+}\left[7 X_{3}+14 X_{1} X_{2}+7 X_{1}^{3}\right]=7 B_{+}\left[X_{3}+2 X_{1} X_{2}+X_{1}^{3}\right]=7 X_{4},
$$

as desired.

Let us now turn to the general case. Let us consider a monomial of degree $r$ contributing in the sum in Eq.(63). It suffices to consider the generic case $s_{1}=\ldots=s_{r}=1$, if 
there are higher powers $s_{i}>1$, the fact that $F_{X_{m}}$ is a derivative ensures that the same argument holds and that the extra $s_{i}$ ! factors in the denominator will be appropriately cancelled.

In the generic case the coefficient of the monomial is $r$ !. Let us first consider the case $r=2$. Contributions to $X_{a} X_{b}$ say, with $a+b=k-m$, are coming from

$$
2 ! F_{X_{m}}\left(B_{+}\left(X_{a+m} X_{b}+X_{a} X_{b+m}\right)\right) .
$$

By assumption of the induction, this delivers, for the non-derivative terms,

$$
2 ![2(k-m)-2] B_{+}\left(X_{a} X_{b}\right),
$$

while from the derivative terms, we get

$$
3 ! B_{+}\left(X_{a} X_{b}\right)
$$

which adds up to

$$
2 ![2((k+1)-m)-1] B_{+}\left(X_{a} X_{b}\right),
$$

as desired. In general, for arbitrary $r$, the simple fact that $r !(r+1)=(r+1)$ ! ensures that each term in the sum homogenously factors $[2((k+1)-m)-1]$, and summing over all monomials and applying the $B_{+}$operator, we get, by Eq. 63) backwards, $F_{X_{m}}\left(X_{k+1}\right)=[2((k+1)-m)-1] X_{k+1-m}$.

Now, let $\sigma_{m}^{n}:=\lim _{\epsilon \rightarrow 0} \epsilon^{n}\left[\phi \circ S \star\left[Y^{n}\right]\right]\left(X_{m}\right)$, with antipode $S$ and grading $Y$ and convolution product $\star$ as in [2].

Then, the procedure DysonScalar is

\section{Prop.2:}

$$
\sigma_{m}^{n}=\sum_{i=1}^{m-1}[2(m-i)-1] \sigma_{i}^{1} \sigma_{m-i}^{n-1} .
$$

Here, $\phi$ is the unrenormalized character on the Hopf algebra $\phi=\left.\Sigma\right|_{q^{2}=1}$. With its help and the Hopf algebra automorphism $\Theta_{\rho}: H \rightarrow H$ of [19],

$$
\Theta_{\rho}(h)=e^{\rho \operatorname{deg}(h)} h, \forall \rho \in H
$$

(deg is the degree: $\operatorname{deg}\left(X_{m}\right)=m$ ) we can write the renormalized character: the character $\Sigma$ is given by

$$
\Sigma\left(q^{2} / \mu^{2}\right)=\phi \circ\left[S \star \Theta_{-\epsilon \log \left(q^{2} / \mu^{2}\right)}\right] .
$$

The Taylor expansion in $\log \left(q^{2} / \mu^{2}\right)$ is then solely a study of the expansion of the Hopf algebra automorphism $S \star \Theta_{-\epsilon \log \left(q^{2} / \mu^{2}\right)}$ which can be written, setting $z=\log \left(q^{2} / \mu^{2}\right)$, as

$$
S \star \Theta_{-\epsilon \log \left(q^{2} / \mu^{2}\right)}=\sum_{i=1}^{\infty} \frac{S \star\left[Y^{i}\right]}{i !}(-\epsilon z)^{i} .
$$

Also, we note that quite generally

$$
\epsilon^{n} \phi\left(S \star Y^{m}\left[X_{j}\right]\right)=\mathcal{O}\left(\epsilon^{n-m}\right), \forall X_{j},
$$


which follows from the fact that $\boldsymbol{\Sigma}$ exists in the limit $\epsilon \rightarrow 0$.

Proof: We use Prop.1 and induction on $m$. We only consider the case $n=2$, the other cases follow identically. The start of the induction involves an explicit check of properties of $\phi\left(X_{1}\right), \phi\left(X_{2}\right)$, which can be done easily using $\phi\left(X_{1}\right)=B_{1}, \phi\left(X_{2}\right)=B_{1} B_{2}$, where

$$
B_{i}=\left.\int d^{D} k \frac{1}{\left[k^{2}\right]^{1+i \epsilon}(k+q)^{2}}\right|_{q^{2}=1} \text {. }
$$

We concentrate on the induction step. Assume

$$
\epsilon^{2} \phi\left[S \star Y^{2}\left[X_{j}\right]\right]=\epsilon^{2} \sum_{i=1}^{j-1} \phi\left[S \star Y\left[X_{1}\right]\right] \phi\left[S \star Y\left[F_{X_{i}}\left(X_{j}\right)\right]\right]
$$

for all $j \leq m$. We want to prove it for $m+1$. Again, we use the presentation Eq.(63) for $X_{m+1}$, for which we write in shorthand $X_{m+1}=B_{+}\left(X_{m}+\sum \prod_{i} X_{i}\right)$, empasizing a decomposition into product terms and the single term $X_{m}$ which is linear in generators in the sum involved in this equation.

We then have

$$
\begin{aligned}
\epsilon^{2} \phi\left(S \star Y^{2}\left[X_{m+1}\right]\right) & =\epsilon^{2} \phi\left[S \star Y^{2}\left[B_{+}\left(X_{m}+\sum \prod_{i} X_{i}\right)\right]\right] \\
& =\epsilon^{2} \phi\left(S \star Y^{2}\left[B_{+}\left(X_{m}\right)\right]\right)+\epsilon^{2} \widetilde{B_{+}} \phi\left(S \star Y^{2}\left[\sum \prod_{i} X_{i}\right]\right) .
\end{aligned}
$$

Here, $\widetilde{B_{+}}(\phi) \equiv \phi \circ B_{+}$and we use

$$
S \star Y^{2} \circ B_{+}=m \circ\left(S \otimes Y^{2}\right) \circ\left(i d \otimes B_{+}\right) \circ \Delta
$$

(as $Y^{2}(e)=0$ and where $m$ is the product, $\Delta$ the coproduct) which further can be written as

$$
m \circ\left(i d \otimes B_{+}\right) \circ\left(S \otimes Y^{2}\right) \circ \Delta,
$$

due to the fact that for the commutator between $Y, B_{+}$we have $\left[Y, B_{+}\right]=B_{+}$. Hence, interchanging $B_{+}$with $Y^{2}$ produces only commutator terms involving $\epsilon^{2} \phi \circ S \star Y^{i}$, with $i=0,1$, which vanish by Eq.(65) in the limit $\epsilon \rightarrow 0$.

Let us treat the two terms on the rhs in the last line of Eq. (66) separately. For the second term, we use that $S \star Y^{k}\left[\prod_{i} X_{i_{j}}\right]=0$ if the product has more than $k$ factors. To see this, consider, for example (using Sweedler's notation),

$$
\begin{aligned}
S \star Y\left[X_{i} X_{j}\right] & =S\left[X_{i}^{\prime} X_{j}^{\prime}\right] X_{i}^{\prime \prime} X_{j}^{\prime \prime}\left[\operatorname{deg}\left(X_{i}^{\prime \prime}\right)+\operatorname{deg}\left(X_{j}^{\prime \prime}\right)\right] \\
& =S \star i d\left(X_{i}\right) S \star Y\left(X_{j}\right)+S \star Y\left(X_{i}\right) S \star i d\left(X_{j}\right)=0,
\end{aligned}
$$

and similarly for higher powers of $Y$. Hence, $S \star Y^{2}$ vanishes on products of more than two arguments, and on two arguments we have, $S \star Y^{2}\left(X_{i} X_{j}\right)=2 S \star Y\left(X_{i}\right) S \star Y\left(X_{j}\right)$. (In general, $S \star Y^{n}$ behaves like a $n$-th derivative acting on a product of functions which vanish at the origin, for example

$$
\left.(f g)^{\prime \prime}=f^{\prime \prime} g+2 f^{\prime} g^{\prime}+f g^{\prime \prime}=2 f^{\prime} g^{\prime} .\right)
$$


Hence, the second term on the rhs above delivers

$$
2 \epsilon^{2} \sum_{i+j=m} \widetilde{B_{+}} \phi\left[S \star Y\left[X_{i}\right]\right] \phi\left[S \star Y\left[X_{j}\right]\right] .
$$

For the first term on the rhs we find

$$
\epsilon^{2}[\phi \otimes \phi]\left(S \otimes Y^{2}\right)\left(i d \otimes B_{+}\right) \Delta\left(X_{m}\right)=m\left[\left(i d \otimes \widetilde{B_{+}}\right)(\phi \otimes \phi)\left(S \otimes Y^{2}\right) \Delta\left(X_{m}\right)\right]
$$

Here we interchanged $B_{+}$with the $Y$ operator as before. The assumption of the induction is

$$
(\phi \otimes \phi) \circ\left(S \star Y^{2}\right) \circ \Delta\left(X_{j}\right)=(\phi \circ S \star Y \otimes \phi \circ S \star Y) \circ \Delta\left(X_{j}\right) .
$$

From this assumption and from Prop.1 we can write for the above expression

$$
\sum_{i}[2(m-i)-1]\left[\phi \circ S \star Y\left(X_{i}\right)\right]\left[\tilde{B}_{+}(\phi) \circ S \star Y\left(X_{m-i}\right)\right] .
$$

Adding the results of the first and second terms for the rhs of (66), we find

$$
\sum_{i}[2((m+1)-i)-1]\left[\phi \circ S \star Y\left(X_{i}\right)\right]\left[\tilde{B}_{+}(\phi) \circ S \star Y\left(X_{m-i}\right)\right] .
$$

Using that $S \star Y$ vanishes on products by Eq.(67), we can combine the resulting expressions to the desired result, by adding zero in form of adding all the product terms in Eq.(63) for $X_{m+1-i}$ to eliminate the $B_{+}$operator in favour of the increased degree $m+1-i$. This proves the proposition.

With these tools, the proof of Theorem 1 becomes a mere comparison of coefficients of two series in an infinite number of variables. Indeed, in both series we can express any appearance of $\sigma_{m}^{n}$ by Prop. 2 as a product $\Pi_{i=1}^{n} \sigma_{m_{i}}^{1}, \sum_{i} m_{i}=m$. Hence, Theorem 1 provides two power series in an infinite set of variables $\sigma_{i}^{1}$. Note that a monomial in degree $m$ in these variables is always accompanied by the factor $z^{m-1}$.

Let us set

$$
\sigma:=\sum_{k=1}^{\infty} \sum_{n=1}^{\infty} \frac{\sigma_{k}^{n} z^{n}}{n !}
$$

and

$$
\sigma^{(i)}:=\sum_{k=1}^{\infty} \sigma_{k}^{i}
$$

Then, Theorem 1 amounts to showing that

$$
\sum_{k=1}^{\infty} \sum_{n=0}^{\infty} \frac{\sigma_{k}^{n+1} z^{n}}{n !} \frac{1}{1-\sigma}=\sum_{m=1}^{\infty} \sigma_{m}^{1} \frac{1}{(1-\sigma)^{2 m}} .
$$

Using Prop.2 and elementary algebra one finds the following coefficients at orders $z^{0}, z^{1}, z^{2} / 2, \ldots$ for the lhs:

$$
z^{0}: \quad \sigma^{(1)}
$$




$$
\begin{array}{ll}
z^{1}: & \sigma^{(1)} \sum_{r_{1}=1}^{\infty}\left(2 r_{1}\right) \sigma_{r_{1}}^{1} \\
\frac{z^{2}}{2}: & \sigma^{(1)} \sum_{r_{1}, r_{2}=1}^{\infty}\left(2 r_{2}\right)\left(2 r_{1}+2 r_{2}\right) \sigma_{r_{1}}^{1} \sigma_{r_{2}}^{1} \\
\ldots & \ldots
\end{array}
$$

which for example implies that the coefficient of the term $\left[\sigma_{1}^{1}\right]^{n} z^{n-1} /(n-1)$ ! is simply $2^{n-1}(n-1)$ !. The crucial step in all this is to recognize how the terms coming from the expansion of $1 /(1-\sigma)$ cancel the ' -1 '-term coming from Prop.2, ie the term $-\sum_{i} \sigma_{i}^{1} \sigma_{m-i}^{n-1}$ in

$$
\sigma_{m}^{n}=\sum_{i}[2(m-i)-1] \sigma_{i}^{1} \sigma_{m-i}^{n-1} .
$$

A similar phenomenon happens in the Taylor expansion of

$$
\frac{1}{\left(1-\sum_{i=1}^{k} \frac{\sigma^{(i)} z^{i}}{i !}\right)^{2 m}}
$$

to the $k$-th order, involved in the rhs of (68). For example, at order $z^{2}, k=2$, the ' -1 'term in $(2 m-1)$ generated by the second derivative does the same job, and in general the lhs and the rhs of (68) give the same series in $z$.

\section{Conclusions}

Returning to the questions in the introduction, we answer them as follows:

1. The non-perturbative problems whose expansions were studied in [1], 2] are defined by $(14,18)$. For the Yukawa problem, a solution in closed form is given by (1), for the anomalous dimension. The duality of Theorem 1 then gives the propagator as a limit of integration of an integral that must evaluate to $\log \left(q^{2} / \mu^{2}\right)$. The particular solution of this duality is given by $(37,38)$, in terms of the complementary error function. The same form of duality applies in the scalar case, though for this we have not yet obtained an explicit solution to the 4th-order equation that results from (53-55). We have, however, found a way of developing the perturbation series almost as efficiently as in the Yukawa case, attaining 500 loops in a few minutes.

2. The obstacle in (5) at $\tilde{\gamma}=-2$, i.e. at coupling $g=2(2 \pi)^{3 / 2} \approx 31.5$ in the Yukawa case, was surmounted, thanks to the parametric non-perturbative solution (1). In the scalar case, the obstacle in (48) at $\gamma=-1$ is surmountable, by numerical solution of the third-order equation for the anomalous dimension. In the Yukawa case, we are able to compute strong-coupling results to very high accuracy and use them as stringent tests of the Borel resummation in [1]; in the scalar case, we have confidence in such strongcoupling resummations, but cannot yet surpass them with the ease afforded by $(1,37,38)$.

Let us now finish this paper by a discussion of the difficulties one confronts in general. Our starting Dyson-Schwinger equations were addressing Feynman graphs representing 
only the Hopf algebra of undecorated rooted trees. To treat the general case, the first observation is that there would be various operators $B_{+}$, indexed by labels corresponding to primitive graphs without subdivergences, of the form

$$
{\widetilde{B_{+}}}^{x}=\frac{a^{\operatorname{deg}(x)}}{\pi^{2 \operatorname{deg}(x)}} \int d^{4} l \frac{F^{x}\left(l^{2}, l \cdot q, q^{2}\right)}{l^{2}},
$$

where deg gives the loop number of the decoration $x, F^{x}$ is some integral kernel obtained from the skeleton graph $x$, and the corresponding integral operator contains a sum $\widetilde{B_{+}}=$ $\sum_{x}{\widetilde{B_{+}}}^{x}$. Here, the previous representation of the $\widetilde{B_{+}}$operator as

$$
\frac{a}{\pi^{2}} \int d^{4} l \frac{q \cdot l}{l^{2}(l+q)^{2}}
$$

would be just the first term in such a series.

A further complication is given by the fact that allowing for internal self-energies and vertex-corrections one would have to consider a coupled system of Dyson-Schwinger equations for those functions. Still, all this is conceptually not too drastic a challenge, and Hopf and Lie algebra structures for the full theory are readily available [3, 18].

But the real difficulty resides in the fact that these new ${\widetilde{B_{+}}}^{x}$ operators offer a choice of as many places as there are internal lines and vertices for the self-energies and vertex corrections to be inserted into $x$. This is in sharp contrast to the Dyson-Schwinger equation studied here which was carefully designed so that it was always the same propagator into which the self-energy was inserted (see Figure 1).

It would be very convenient if the characters of the full Hopf algebra of Feynman graphs would turn out to be independent of such choices, alas, in dimensional regularization, they are not. Nevertheless, they fail to be so in a most interesting way, as mentioned in [20]: only lower order pole terms are affected by such choices, and a proper understanding of the operad structure of Feynman graphs [20] should ultimately allow to generalize the methods presented here to the full theory.

\section{References}

[1] D.J. Broadhurst, D. Kreimer, Phys. Lett. B475 (200) 63, hep-th/9912093.

[2] D.J. Broadhurst, D. Kreimer, J. Symb. Comput. 27 (1999) 581, hep-th/9810087.

[3] D. Kreimer, Adv. Theor. Math. Phys. 2 (1998) 303, q-alg/9707029.

[4] A. Connes, D. Kreimer, Comm. Math. Phys. 199 (1998) 203, hep-th/9808042.

[5] D. Kreimer, Comm. Math. Phys. 204 (1999) 669, hep-th/9810022.

[6] D. Kreimer, Adv. Theor. Math. Phys. 3 (1999) 327, q-alg/9901099.

[7] R. Delbourgo, A. Kalloniatis, G. Thompson, Phys. Rev. D54 (1996) 5373, hep-th/9605107. 
[8] R. Delbourgo, D. Elliott, D.S. McAnally, Phys. Rev. D55 (1997) 5230, hep-th/9611150.

[9] D.J. Broadhurst, Z. Phys. C58 (1993) 339.

[10] D.J. Broadhurst, A.G. Grozin, Phys. Rev. D52 (1995) 4082, hep-ph/9410240.

[11] U. D. Jentschura, J. Becher, E. J. Weniger and G. Soff, Phys. Rev. Lett. 85 (2000) 2446 hep-ph/9911265];

U. D. Jentschura, Phys. Rev. D 62 (2000) 076001 hep-ph/0001135;

U. D. Jentschura and G. Soff, Improved conformal mapping of the Borel plane, hep-ph/0006089.

[12] N.N. Bogoliubov, O. Parasiuk, Acta Math. 97 (1957) 227;

K. Hepp, Comm. Math. Phys. 2 (1966) 301;

W. Zimmermann, Comm. Math. Phys. 15 (1969) 208.

[13] D. Kreimer, J. Knot Th. Ram. 6 (1997) 479, q-alg/9607022.

[14] D. Kreimer, R. Delbourgo, Phys. Rev. D60 (1999) 105025, hep-th/9903249.

[15] A. Connes, D. Kreimer, JHEP 9909 (1999) 024, hep-th/9909126.

[16] A.C. Hearn, REDUCE User's Manual, Version 3.7, March 1999.

[17] D.H. Bailey, ACM Transact. Math. Softw. 19 no.3 (1993) 288; 21 no.4 (1995) 379.

[18] A. Connes, D. Kreimer, Commun. Math. Phys. 210 (2000) 249, hep-th/9912092.

[19] A. Connes, D. Kreimer, hep-th/0003188, to appear in Commun. Math. Physics.

[20] D. Kreimer, hep-th/0010059, to appear in Phys. Reports.

[21] D.J. Broadhurst, D. Kreimer, hep-th/0001202, to appear in Commun. Math. Physics. 
Table 1 Coefficient of the scalar perturbation series with 1675 digits at 500 loops

G500 $=206261451966080541451119356265266407905816117576895601520616328670543304097$ 62369668214104674763068056454522518617422020409397336434904863988900797769773644 47129884863324773181376863120291798830884688213932683869821267125662274428136514 68974978228592824043044373847281757207937081063432528806815509319762088807291996 54549245884853496719417048678199825379018355919198123075612308008976364608893906 00835837012056033720017238115336850340799075684336975651857656078799282745256216 85768456030809283727097722850488278232311177219444745322287340871435443707536590 64304859950724683157717734493071321199539578218428617617722892100276682781401203 04983974209704793621909710059353724523231635766062166284812903992269403282699432 81718327508638643305481989940132234093616573076862094588977827344981584305605437 66475002382217933275761312682929603923397580260987048907414858143897114762331252 08694985337972553885925402003826420205441859988844001867088083850782378303677991 14077650584544145709672328391394562704209221732180879565868213522109303655045186 92714017665002971967455255310508358729281544729403249398746232320441525286283859 23093041626365262630100048817481274793707664791767175677240144896307853488347045 21622394885797995125083750860330519417878429051836575477220881369445751634601965 33191009573619480068718080810533581305996863996579338522874547127421808710757882 86996199556804886954946559116947132125235605586627322129268965041445488085748194 82341875039156647569797757032552836429751077302524927736861138479038542006096835 73747720303607608007740173613335602076396299832459826245418033598839559699294537 37336134624690115674194793212055897162647586497730033948880084738561472545509216 